\title{
Towards Inductive Generalisation in Higher Order Logic
}

\author{
Cao Feng, Stephen Muggleton \\ The Turing Institute, George House, 36 North Hanover Street; \\ Glasgow G1 2AD, UK
}

\begin{abstract}
In many cases, higher order (Horn) clauses are more suited to express certain concepts when relations between predicates exist. However, to date there has been no appropriate higher order formalism within which efficient inductive generalisation can be carried out. This paper describes inductive generalisation in $M_{\lambda}-$ a higher order formalism which not only retains the expressiveness of $\lambda$-calculus but also provides for effective and efficient inductive generalisation. The main strength of $R_{\lambda}$ is twofold: it is a higher formalism extension of the (clausal) first order logic and it can be mechanised in a way similar to the first order case in Horn clause form. For a class of restricted $M_{\lambda}$, their least general generalisation (LGG) is unique, and so is their most general unification. Inductive generalisation in $M_{\lambda}$ is implemented in the algorithm HOLGG. This algorithm has been applied to some interesting induction problems in the induction of higher order rule templates and automatic program transformation.
\end{abstract}

\section{Introduction}

Generalisation forms the basis of most inductive learning systems. In first order logic, generalisation has been well-understood [Plotkin1970, Plotkin1971, Reynolds1970], and many algorithms have been devised based on these principles [Muggleton and Feng1990]. Using a first order inductive tool [Muggleton and Feng1990], we can obtain the clauses:

$\forall X \forall Y \forall Z$.ancestor $(X, Y) \leftarrow$

$$
\begin{aligned}
& \text { ancestor }(X, Z), \text { ancestor }(Z, Y) . \\
& \forall X \forall Y \forall Z \text {.less_than }(X, Y) \leftarrow \\
& \text { less_than }(X, Z), \text { less_than }(Z, Y) .
\end{aligned}
$$

which are the generalisation respectively of the facts less_than $(1,3)$, less_than $(2,4), \ldots$ and the facts ancestor (john, steve), ancestor(steve, mike), ...

The clauses in (1) and (2) are very similar in the sense that (1) can be obtained from (2) by exchanging the predicate symbol ancestor with less_than in all its appearances, and vice versa. If we allow for a special variable $P$, it is not difficult to see that both clauses in (1) and (2) are "substitution instances" of the clause:

$$
\begin{array}{r}
\forall X \forall Y \forall Z . P(X, Y) \leftarrow \\
P(X, Z), P(Z, Y) .
\end{array}
$$

with $P$ being substituted by ancestor for (1) and less_than for (2). As $P$ is a predicate variable, this clause needs a higher order language that goes beyond the first order predicate logic used in present machine learning research and applications.

In general, a term $E$ is inore general than another term $F$ whenever there is a substitution $\theta$ for which $E \theta=F$. This is normally called term $\theta$-subsumption. Obviously formula (3) is more general than both (1) and (2). Because the clause in (3) contains the higher order variable " $P$ ", this generality cannot be understood under its meaning in first order logic. It has to be made precise by formal semantic and syntact tic definitions in higher order logic. Thus we needto. consider the problem of how $P, X, Y$ and $Z$ are in terpreted in (1), (2) and (3). This has been partially tackled by logic programming in higher order Horn clauses [Nadathur and Miller1990], which is an exten sion of (first order) logic programming methods to deal with higher-order terms.

Obviously, the higher order clause is a more powerifil representation both in terms of expressiveness and ficiency. Conversely, if we are given the higher order 
clause such as the clause in (3) we hope that we can more quickly find the rules (1) and (2) with respect to some given facts (perhaps in higher order).

\section{Motivation}

In order to introduce a higher order language we need to address several issues pertinent in inductive learning. The main concerns are:

- Expressiveness. Meta-level information about inductive problems can, in many cases, only be expressed adequately with higher order terms. The induction of program transformation rules in [Huet and Lang1978] is a case in point.

- Induction. Inasmuch machine learning is concerned, we need to consider how (3) can be obtained, especially from the given formulae such as (1) and (2). This gives rise to the need of the induction of (3). Similar to the case in first-order logic [Plotkin1970, Plotkin1971, Muggleton and Feng1990], this may be solved by generating the least general generalisation (LGG) of terms in this language.

- Efficiency. To achieve practical efficiency, deduction and induction in this language must be easy to compute. In specific, corresponding to first order logic the lower bound of generalisations and unifications of terms must be unique as both are unique for the language of first order terms.

Following Robinson, unification has become the basis of resolution-based logic deduction. Huet [Huet1975] found a semi-deterministic unification algorithm for higher-order terms. Inspired by the success of the logic programming language Prolog, $\lambda$ Prolog [Nadathur and Miller1990] has been developed as a full higher-order extension of Prolog. For example, the formula (3) is a $\lambda$ Prolog clause. $\lambda$ Prolog has been successfully applied in writing program transformers, theorem provers and a number of other areas [Paulson1986, Dietzen and Pfenning1989].

Similar to most general unification (MGU), the least general generalisation (LGG) of terms [Plotkin1970, Muggleton and Feng1990] plays an important role in the emerging field of inductive logic programming, which is evolved from logic programming and conventional machine learning [Muggleton1990]. However, as the field matures the need for various higherorder notions has arisen in order to guide the induction of Horn clauses from facts (ground atoms). These include rule templates [Raedt and Bruyonooghe1990,
Kietz and Wrobelto appear, Harao1990], predicate determinations

and modes [Muggleton and Feng1990], variable types [Lavrac and Dzeroski1991, Quinlan1990] and predicate commutativity [Lavrac and Dzeroski1991].

At present, higher order terms already have many applications in machine learning: these include inductive learning [Muggleton and Feng1990], analogical reasoning [Harao1990], constructive induction [Raedt and Bruyonooghe1990] and model-driven induction [Kietz and Wrobelto appear]. It is argued that higher-order terms provide elegant expressions for many problems. However, the higher order formalisms used in research to date are unsatisfactory and lack a sound semantics. In particular they deliberately avoid using $\lambda$-calculus. They therefore lose the expressive power of higher-order logic.

In a higher order logic, a term $E$ is more general than another term $F$, or $F$ is more specific than $E$, if and only if there is a substitution $\theta$ such that $E \theta$ is $\lambda$ convertible to $F$. This is denoted by $E \geq_{\theta} F$ or $F \leq_{\theta}$ $E$. The relation $\geq_{\theta}$ is transitive, nonsymmetric and reflexive and thus defines a partial ordering over terms. $E={ }_{\theta} F$, or $E$ is $\theta$-equal to $F$ if and only if $E \geq_{\theta} F$ and $F \geq_{\theta} E$. When $E \geq_{\theta} F$ but $E \neq_{\theta} F$, we say $E>_{\theta} F$ or $F<_{\theta} E$.

A term $E$ is a common generalisation of a set of terms $\mathcal{T}$ if and only if $E$ is more general than each of the terms in $\mathcal{T}$. A term $E$ is a least general generalisation of a finite set of terms $\mathcal{T}$ if and only if: 1) $E$ is a common generalisation of $\mathcal{T}$; and 2) for any $F<{ }_{\theta} E$, $F$ is not a common generalisation of $\mathcal{T}$.

It is shown in [Feng and Muggleton1991] that in general there are multiple or an infinite number of solutions for the LGG of two higher-order terms that are expressed in full $\alpha \beta \eta \lambda$-calculus (see Appendix A). In order to compute LGGs efficiently, it is desirable to have a restricted $\lambda$ calculus such that MGU and LGG are unique in the condition of not sacrificing effectiveness. It is, indeed, only the formulae similar to (3) in Section 1 that we are most interested in. To achieve this we will have to place some additional conditions on $\lambda$-calculus and hopefully we can find a manageable subclass of the general $\lambda$-calculus. One such restriction is $L_{\lambda}$ [Miller 1990]. $L_{\lambda}$ [Miller1990, Pfenning1991] is a restricted subset of $\lambda$ Prolog which has unique $\mathrm{MGU}$ and LGG. But it is ill-equipped to express many problems. In particular it cannot represent recursions in its terms which we shall see in Section 6. 


\section{$3 \quad M_{\lambda}$ : a restricted higher order language}

Readers unfamiliar with simple (typed) $\lambda$-calculus may go to the Appendix A for the relevant information on $\lambda$-calculus. Throughout this paper, the terminology is similar to that used in logic programming, especially Prolog. Variables are denoted by upper case letters $U$ to $Z$; formulae and terms by other upper case letters; constants by lower case letters; types by Greek letters $\alpha, \beta$ and $\gamma$; and substitutions by Greek letters $\theta, \delta$ and etc., when confusion does not arise. The constant " $\leftarrow$ " is often used as an infix operator for convenience of understanding. In a few occasions we will use calligraphical upper-case letters to denote sets. Suitable super- or sub-scripts may also be used.

A $L_{\lambda}$ [Miller1990] term contains only free variables that are applied to bound variables. For example, the formula

$$
\begin{array}{r}
\lambda X . \lambda Y . \lambda Z .(P(X, Y) \leftarrow \\
P(X, Z), P(Z, Y)) .
\end{array}
$$

is a $L_{\lambda}$ term. The free variable $P$ only has arguments that are bound variables in the formula. $P$ is said to be applied to $X$ and $Y$ and $\lambda X$ is called an abstraction from $\lambda Y . \lambda Z .(P(X, Y) \leftarrow P(X, Z), P(Z, Y))$. A variable in an abstraction is called a bound variable, otherwise it is a free variable. We will also say a variable is free to a term (or subterm) if it is not bound by that term (or subterm). When there are many successive abstraction variables in a formula such as in (4) we may write " $\lambda X Y Z$ ".

The term in (4) is closely related to (3) as the quantifier " $\forall X . F$ " is an abbreviation for " $\Pi(\lambda X . F)$ " and $I$ is a constant expressing universal quantification (and similarly " $\exists X . F$ " is for " $\Sigma(\lambda X . F)$ ").

Though MGU and LGG is unique in $L_{\lambda}$, it is too constrained to express simple terms that contain recursion. Practical examples of the restrictions of $L_{\lambda}$ can be seen in Section 6 . But now let us look at a simple modification of the above term:

$$
\begin{gathered}
\lambda X Y Z .(P(s(X), Y) \leftarrow \\
P(X, Z), P(Z, s(Y))) .
\end{gathered}
$$

Because it contains a constant $s$ in the arguments of $P$, (5) is not a $L_{\lambda}$ term which is important in many logic programming problems (see Section 6). We shall introduce some extra-logical extensions to $\lambda$ Prolog. This will be called $M_{\lambda}$. In this calculus, a subterm such as $s(X)$, called an object term, is explicitly allowed.

An object term consists of externally bound variables and constants of distinct types from any bound variables without abstraction. A variable $X$ in a term $F$ is externally bound if $F$ is a subterm of some term $E$ and $X$ is bound by an abstraction in $E$ outside of $F$. The use of object terms gives rise to the necessity of the other extensions in $M_{\lambda}$.

$M_{\lambda}$ is a restricted typed $\lambda$-calculus. In $\left.M_{\lambda}: 1\right)$ It is allowed to perform $\alpha, \beta, \delta_{0}$ and $\eta$ conversions on terms. The $\delta_{0}$ conversion rule is described in Appendix B; 2) Any free variables in $M_{\lambda}$ terms are only applied to arguments that are object terms; 3) It contains at least constants $\chi$ and $\psi$ such that for any object term of $M_{\lambda}, E, \chi(E)=E_{2}$ and $\psi(E)=E_{1}$ if $E=\left(E_{1} E_{2}\right)$, and $\psi(E)=\chi(E)=E$ otherwise. (Both are undefined for non-object terms).

Clearly $M_{\lambda}$ is an extension of the $L_{\lambda}$ language [Miller1990], in which the $\delta_{0}$ rule is not permitted and the arguments of free variable functions can only be externally bound variables. $\chi$ and $\psi$ are analytical selectors in an analytical syntax as defined by McCarthy. Fortunately many programming languages, such as Lisp and Prolog, have these functions. In $M_{\lambda}$ these are further restricted to the application to object terms, which do not contain abstractions as defined in this paper. Also note these extensions are introduce constants into the formalism and therefore are extralogical.

\section{$4 \mathrm{M}_{\lambda}$ normal and nonredundant terms}

A term is $M_{\lambda}$ normal if and only if it is in $\alpha \beta \delta_{0} \eta$ normal form and contains no irreducible $\delta_{0}$ expressions. We will be interested in LGGs and MGIs that are $M_{\lambda}$ normal. The following definition is adopted from that in Section 2. $E$ is the $M_{\lambda}$ normal $L G G$ of a set of $M_{\lambda}$ normal terms $\mathcal{T}$, if and only if: 1) $E$ is $M_{\lambda}$ normal and is also a common generalisation of $\mathcal{T}$; and 2) $F$ is not a common generalisation of $\mathcal{T}$ for any $M_{\lambda}$ normal term $F<_{\theta} E$.

For $M_{\lambda}$ normal term $S=p(U, U(a))$, the substitution containing $\delta_{0}$ conversions such as $\theta=$ $\left\{U / \lambda X\right.$.if $X=a$ then $E_{1}$ else $\left.E_{2}\right\}$ is not applicable [Curry et al.1958]. If applied it results in $S \theta=$ $p\left(\left(\lambda X\right.\right.$.if $X=$ a then $E_{1}$ else $\left.\left.E_{2}\right), E_{1}\right)$, which cannot be reduced further because $X$ in $X=a$ is a free variable to $X$. Thus this term is not a $M_{\lambda}$ normal term.

Let $E$ be a $M_{\lambda}$ term and $E^{\prime}$ be a $\theta$-equal term to $E$. A subterm $F$ of $E$ will have some trace $F^{\prime}$, so to speak, in $E^{\prime} . F^{\prime}$ is called the residual of $F$. A subterm $F$ of $E$ is redundant if $E^{\prime \prime}=E^{\prime}-\left\{F^{\prime}\right\}$ and $E^{\prime \prime}=_{\theta} E, \mathrm{i}$, the residual of $F$ can be removed and still maintain $\theta$-equality.

Clearly, any redundancy can only happen in terms 
with free variable functors. If $E$ is a subterm of $F$ with free variable functor and no other subterm of $F$ has the same functor, then any term containing only constants and repetitive bound variables in $E$ is redundant: $\mathrm{Be}-$ cause $E$ is the only subterm that has the free variable as its functor, we are free to devise various substitutions to decompose $E$ into its subterms and then remove all constants and repetitive bound variables from it. The operation to remove redundant subterms is called a reduction (not to be confused with $\alpha, \beta, \delta_{0}$ and $\eta$ reductions of $\lambda$ calculus). After simple reductions, constants will appear only in subterms that appear in multiple places of a terms. [Feng and Muggleton1991] describes an algorithm that can reduce a $M_{\lambda}$ to its nonredundant form. In the rest of the paper we refer terms to $M_{\lambda}$ normal, nonredundant terms and their LGGs to $M_{\lambda}$ normal, nonredundant LGGs.

We need also to consider unification, which is the dual of LGG. $E$ is the common instance of a set of $M_{\lambda}$ terms $T$ if $E$ is more specific than each element of $\mathcal{T}$. $E$ is the most general instance (MGI) of a set of $M_{\lambda}$ terms $\mathcal{T}$ if and only if: 1) $E$ is the common instance of $\mathcal{T}$; and 2) $F$ is not the common instance of $\mathcal{T}$ for any $F>_{\theta} E$. The substitution $\theta$ for which $E_{i} \theta=E, \forall E_{i} \in \mathcal{T}$ is called the most general unifier (MGU). $M_{\lambda}$ normal MGU is similarly defined except that $E, F$ and $E_{i} \in \mathcal{T}$ are all $M_{\lambda}$ normal terms. It is proved in [Feng and Muggleton1991], in normal and nonredundant form the MGU and LGG of $M_{\lambda}$ terms are unique. The unification algorithm is also given in [Feng and Muggleton1991].

\section{Implementation}

LGG and unification in $M_{\lambda}$ is implemented in Prolog, and is called HOLGG. The LGG algorithm has two parses. The first parse is ELGG which collects all the multiple appearances of subterms into a set of triples $\Gamma=\left\{\left(\tilde{X}, S_{1}, T_{1}\right\rangle\right\}$. Then $\Gamma$ is sorted to obtain $\Gamma=$ $\bigcup_{i=1}^{m} \Gamma_{i}$ for which $\Gamma_{i}=\left\{\left\langle\tilde{X}, S_{j}, T_{j}\right\rangle\right\}$ and each $S_{j 1}$ and $S_{j 2}$ in $\Gamma_{i}$ have the same functor and so do $T_{j 1}$ and $T_{j 2}$. In the second parse, CLGG takes $S, T$ and $\Gamma_{i}(i=$ $1, \ldots, m)$ as input and produces $F$. CLGG also calls the algorithm VLGG.

MLGG: anti-unification (LGG) algorithm for $M_{\lambda}$ normal terms.

INPUT: two $M_{\lambda}$ normal terms $S$ and $T$ of the same type;

OUTPUT: a $M_{\lambda}$ normal term $F=$

$M L G G(S, T)$.

1. $\Gamma=E L G G(S, T, \emptyset)$;

2. Sort $\Gamma$ such that $\Gamma=\bigcup_{i=1}^{n} \Gamma_{i}$ for which
$\Gamma_{i}=\left\{\left(\tilde{X}, S_{i}\left(S_{j 1}, \ldots, S_{j n}\right), T_{i}\left(T_{j 1}, \ldots, s_{j k}\right)\right\rangle\right\}$

for $i=1, \ldots, m$;

3. $F=C L G G(S, T, \Gamma, \emptyset)$.

\section{ELGG}

INPUT: $S, T$ and the binding variables $\tilde{X}$; OUTPUT: $\Gamma=\left\{\left\langle F_{p}, S_{p}, T_{p}\right\rangle\right\}$ where $F_{p}, S_{p}$ have different functors.

1. If $S=\lambda \tilde{Y} . S_{1}$ and $T=\lambda \tilde{Y} . T_{1}$, then $\Gamma=$ $\operatorname{ELGG}\left(S_{1}, T_{1}, \tilde{X} \tilde{Y}\right)$

2. If $S=S_{0}\left(S_{1}, \ldots, S_{n}\right), T=T_{0}\left(T_{1}, \ldots, T_{n}\right)$ and $S_{0}=T_{0}=C$, where $C$ is a constant or $C \in \tilde{X}$, then $\Gamma=\bigcup_{i=1}^{n} E L G G\left(S_{i}, T_{i}, \tilde{X}\right)$;

3. If $S_{0} \neq T_{0}$, then $\Gamma=\{\langle\tilde{X}, S, T\rangle\}$.

\section{CLGG}

INPUT: $S, T$, the sorted $\Gamma$ and the binding variables $\tilde{X}$;

OUTPUT: $F$ is the $M_{\lambda}$ normal LGG of $S$ and $T$.

1. If $S=\lambda \tilde{Y} . S_{1}$ and $T=\lambda \tilde{Y} . T_{1}, F=$ $\lambda \tilde{Y} . C L G G\left(S_{1}, T_{1}, \Gamma, \tilde{X} \tilde{Y}\right)$

2. If $S=S_{0}\left(S_{1}, \ldots, S_{n}\right), T=T_{0}\left(T_{1}, \ldots, T_{n}\right)$ and $S_{0}=T_{0}$, then $F=C\left(F_{1}, \ldots, F_{n}\right)$ where $F_{i}=$ $C L G G\left(S_{i}, T_{i}, \Gamma, \tilde{X}\right)$;

3. If $S=S_{0}\left(S_{1}, \ldots, S_{n}\right), T=T_{0}\left(T_{1}, \ldots, T_{n}\right)$ and $S_{0} \neq T_{0}$, and $\langle\tilde{X}, S, T\rangle$ is in $\Gamma_{i}$ and there exist $F_{i}(i=1, \ldots, l)$,

3.1. If $S_{0}$ and $T_{0}$ are constants or free variables, $F=V_{S_{0}, T_{0}}\left(F_{1}, F_{2}, \ldots, F_{l}\right)$;

3.2. If $S_{0} \in \tilde{X}$ and $T_{0}$ is a constant or a free variable, $F=V_{S_{0}, T_{0}}\left(S_{0}, F_{1}, F_{2}, \ldots, F_{7}\right)$;

3.3. If $S_{0}$ is a constant or a free variable and $T_{0} \in \tilde{X}$, then $F=$ $V_{S_{0}, T_{0}}\left(T_{0}, F_{1}, F_{2}, \ldots, F_{1}\right)$

3.4. If $S_{0} \in \tilde{X}$ and $T_{0} \in \tilde{X}$, then $F=$ $V_{S_{0}, T_{0}}\left(S_{0}, T_{0}, F_{1}, F_{2}, \ldots, F_{l}\right)$; where $F_{S}$ $=V L G G\left(S_{i 1}, V L G G(\ldots, V L G G\right.$ $\left.\left.\left(S_{i, j-1}, S_{i j}, \tilde{X}\right), \ldots\right), \tilde{X}\right)$ and $F_{T}=$ $V L G G\left(T_{i 1}, V L G G\left(\ldots, V L G G\left(T_{i, j-1}\right.\right.\right.$, $\left.\left.\left.T_{i j}, \tilde{X}\right), \ldots\right), \tilde{X}\right)$ so $S=\left(\ldots\left(\left(\lambda \tilde{X} . F_{S}\right) F_{1}\right)\right.$ $\left.\ldots F_{l}\right)$ and $T=\left(\ldots\left(\left(\lambda \tilde{X} . F_{T}\right) F_{1}\right) \ldots F_{l}\right)$;

4. If $S=S_{0}\left(S_{1}, \ldots, S_{n}\right), T=T_{0}\left(T_{1}, \ldots, T_{n}\right)$ and $S_{0} \neq T_{0}$,

4.1. If $S_{0}$ and $T_{0}$ are constants or free variables, $F=V_{S_{0}, T_{0}}\left(X_{1}, X_{2}, \ldots, X_{l}\right)$;

4.2. If $S_{0} \in \tilde{X}$ and $T_{0}$ is a constant or free variable, $F=V_{S_{0}, T_{0}}\left(S_{0}, X_{1}, X_{2}, \ldots, X_{l}\right)$;

4.3. If $S_{0}$ is a constant or a free variable and $T_{0} \in \tilde{X}$, then $F=V_{S_{0}, T_{0}}\left(T_{0}, X_{1}\right.$, $\left.X_{2}, \ldots, X_{l}\right)$;

4.4. If $S_{0} \in \tilde{X}$ and $T_{0} \in \tilde{X}, F=V_{S_{0}, T_{0}}$ $\left(S_{0}, T_{0}, X_{1}, X_{2}, \ldots, X_{l}\right)$; where $X_{i} \in \tilde{X}$ 
$(i=1, \ldots, l)$ are bound variables in $S$ and $T$.

VLGG: Variablisation of terms in $M_{\lambda}$.

INPUT: two terms $S$ and $T$ and binding variables $\tilde{X}$;

OUTPUT: $F=V L G G(S, T, \tilde{X})$.

1. If $S=\lambda \tilde{Y} . S_{1}$ and $T=\lambda \tilde{Y} . T_{1}$, then $F=$ $\lambda \tilde{Y} . V L G G\left(S_{1}, T_{1}, \tilde{X} \tilde{Y}\right)$

2. If $S=T=C$ and $C$ is a constant or $C \in$ $\tilde{X}$, then $F=C$;

3. If $S=C\left(S_{1}, \ldots, S_{n}\right)$ and $T=C\left(T_{1}, \ldots, T_{n}\right)$ and $C$ is a constant or $C \in \tilde{X}$, then $F=$ $C\left(V L G G\left(S_{1}, T_{1}, \tilde{X}\right), \ldots, V L G G\left(S_{n}, T_{n}, \tilde{X}\right)\right)$;

4. If $S, T$ contain variables in $\tilde{X}$, then fail otherwise $F=V_{S, T}$ for a variable named by $S$ and $T$.

\section{Applications}

LGG has played an important role in inductive logic programming in first-order logic. The following examples show the applications of LGG to acquire higherorder clause templates from given first-order clauses.

Example 1. Given the first-order facts:

less_than $(0,2)$, less_than $(1,3), \ldots$

less_than $(0,3)$, less_than $(1,4), \ldots$

less_than $(0,1)$, less_than $(1,2), \ldots$

we are able to obtain the following, using an algorithm such as Golem [Muggleton and Feng1990]:

$\forall X Y Z$.less_than $(X, Y) \leftarrow$ less_than $(X, Z)$, less_than $(Z, Y)$,

and similarly

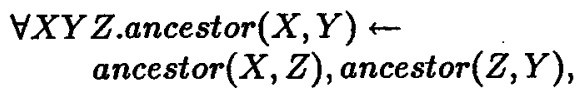

In both clauses $X, Y$ and $Z$ are universally quantified. Note $\forall X . F$ is the abbreviation of $\Pi(\lambda X . F)$ and $\Pi$ expresses universal quantification. Their higher-order LGG is

$\forall X Y Z . P(X, Y) \leftarrow P(X, Z), P(Y, Y)$,

where $P$ is a free variable and "๘" is an infix constant. $P$ may then become universally quantified as this generalisation is accepted.

Though $L_{\lambda}$ can still be used to express the higher-order term in Example 1, the clause in the following example, which contains recursion and represents a majority of problems that we are interested in, cannot be expressed within $L_{\lambda}$. This is because $L_{\lambda}$ forbids the existence of constants in the arguments of free variables.

Example 2. Given the first-order clauses with $X, Y$, $Z$ and $W$ universally quantified,

$$
\begin{aligned}
& \forall X Y Z W . r e v e r s e(\operatorname{cons}(X, Y), Z) \leftarrow \\
& \quad \text { reverse }(Y, W), \\
& \quad \text { append }(W, \text { cons }(X, n i l), Z) . \\
& \forall X Y Z W \text {.insert_sort }(\operatorname{cons}(X, Y), Z) \leftarrow \\
& \quad \text { insert_sort }(Y, W), \text { insert }(X, W, Z) .
\end{aligned}
$$

we can obtain

$$
\begin{gathered}
\forall X Y Z W . P(\operatorname{cons}(X, Y), Z) \leftarrow \\
P(Y, W), Q(W, X, Z) .
\end{gathered}
$$

where "reverse" is a version often referred to as "naive reverse", $P$ and $Q$ are free variables, and "cons" is a list processing function.

One may observe that the first-order facts seem to be the objects in the induction of first-order clauses. These clauses then become objects that characterise the properties of higher-order objects - in this particular case it is second order predicate constants such as "reverse" and "insert". At this "order", we are mainly concerned with the properties of the predicates, the first order objects will be universally quantified, and they are "taken for granted" when studying objects that may apply on them. After this, the higherorder objects may become universally quantified.

If we extend this scenario further, we can imagine that through progressive quantification clauses of successive orders can be induced that characterise objects of higher-order objects.

Another application area is the discovery of program transformation rules. Huet and Lang [Huet and Lang1978] discussed methods for program transformation of recursive computations into iterative ones. A set of second order clause templates for transformation were suggested and they are applied through second order unification to produce more efficient programs based on the Darlington and Burstall [Burstall and Darlington1977] method. As they remarked, the opposite problem with regarding to the discovery of such templates is a difficult task. Few templates are known and no automatic methods exist for performing such discovery.

Example 3. The higher-order logic clause in Example 1 , though interesting, is computationally inefficient To satisfy the first predicate $P(X, Y)$, it needs to non deterministically satisfy $P(X, Z)$ and then $P(Z, Y$ More efficient programs for ancestor and less_than ar 
respectively:

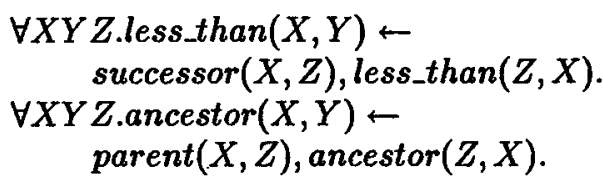

where successor $(X, Z)$ expresses that $Z$ is the successor of $X$ in Peano's formalism, and parent $(X, Z)$ states that $Z$ is the parent of $X$. Both are computational more efficient. Thus possible program transformations are:

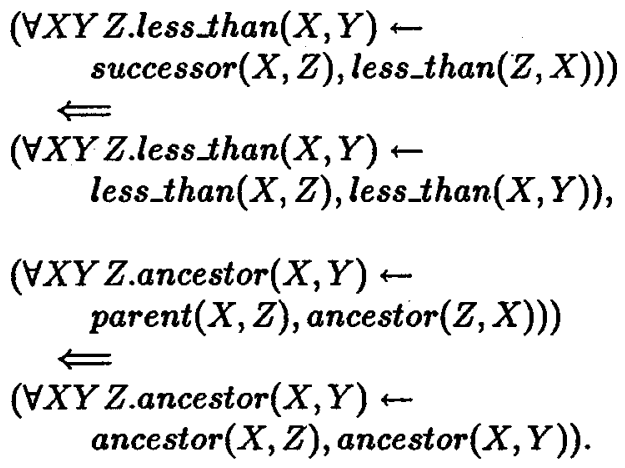

The LGG produces a program transformation template, though the conditions for the transformation are omitted.

$$
\begin{aligned}
& (\forall X Y Z . P(X, Y) \leftarrow Q(X, Z), P(Z, X))) \\
& (\forall X Y Z . P(X, Y) \leftarrow P(X, Z), P(X, Y)) .
\end{aligned}
$$

In fact, such a template is applicable when $Q$ is a special case of $P$ (i.e. $P$ by one).

Example 4. The recursive list reverse program is described in 3. A more efficient iterative (tail recursive) version of it is:

\section{$\forall X Y Z W U$.reverse1(cons $(X, Y), Z, W) \leftarrow$ append $([X], Z, U)$, reverse $1(Y, U, W))$}

where "reverse 1 " contains an accumulator $Z$. When "reverse1" starts with "reverse1(List, nil,

ReversedList)" and is terminated by "reverse1(nil, ReversedList, ReversedList)", it yields the reversed list. Thus we have a transformation

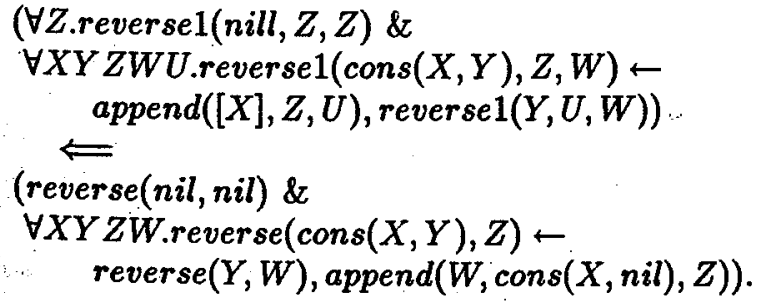

We also know another transformation which concerns with the addition of the elements in a list:

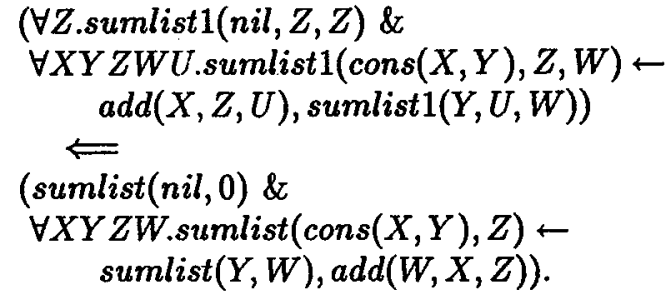

where "plus" is a function that returns the addition of two numbers. When started with "sumlist 1 (List, 0, SumOfList)" and terminated by "sumlist1(nil, SumOfList, SumOfList)", the iterative computation also returns the sum of the elements in the list. The LGG of the two is:

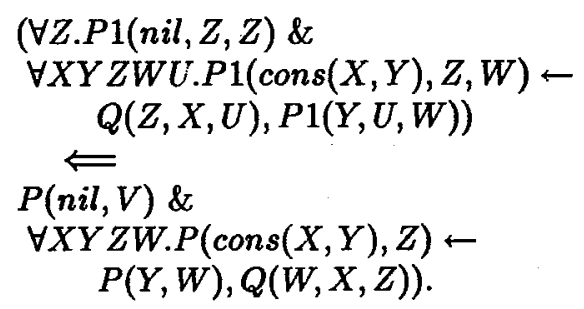

with free variables $P, Q, P 1$ and $V$.

This is an alternative expression of McCarthy's transformation [McCarthy 1960]. For the sake of convenience we have omitted the conditions for this transformation to apply. It is in fact that, among others, $V$ must be the lower bound element of the appropriate type and $Q$ be a transitive and communicative function. This problem can be addressed by relative least general generalisation (RLGG) that will be discussed briefly in Section 7. However, its detail is beyond the scope of this paper.

The other potential application areas are analogical reasoning and the automatic acquisition of grammar rules from example sentences and the generalisation of proofs. However we will not discuss them in this paper.

\section{Conclusion and future research directions}

Recently, inductive logic programming [Muggleton1990] has witnessed a growing trend in utilising higher-order (or meta-level) logical notions in existing ILP framework. This is motivated, in part, by the need to develop more effective and efficient ILP methods. These notions are often adopted as declarative biases in many forms including functional constraints on the predicates in clauses and 
templates for the clauses being induced. However current methods lack a coherent framework for accommodating these notions. In this paper, we found a class of higher order terms that are sufficiently expressive and still have unique MGI and LGG. Their generalisations also proved to be computationally efficient. This can be demonstrated by the various applications in Section 6 . This lays the foundation for studying the relative least general generalisation of such terms.

Our future research work is concerned with expanding the current (first order) ILP framework, in which higher-order inductive inference may be described as the discovery of a hypothesis $H$ from examples and background knowledge such that:

$$
\begin{aligned}
& M \wedge B \wedge H \vdash E^{+} \\
& M \wedge B \wedge H \forall E^{-}
\end{aligned}
$$

if $M \wedge B \nvdash E^{+} . M$ in relation (6) represents a set of higher-order ( $\lambda$ Prolog) clauses. The hypotheses in $H$ now can be either first-order or higher-order clauses. $B$ is the background knowledge, $E^{+}$and $E^{-}$are respectively the set of positive and negative examples. Corresponding to existing ILP theory, it is necessary to develop methods for generalisation in higher-order logic. In doing so we hope to achieve two aims: a) to develop more efficient methods for inducing firstorder clauses, and b) to induce higher-order clauses such that they can aid induction of both classes of logic programs.

From the experience of ILP in first-order logic, in the next step we need to study the relative least general generalisation (RLGG) for higher-order logic programs in the presence of background knowledge. Similar to the first-order case [Muggleton and Feng1990], we may have to deal with a restricted logical model of the background knowledge. We also need to investigate the computability of such a model of the background knowledge. If this is successful the results will have implications in ILP and to the discovery of automatic program transformation techniques.

Acknowledgements. The authors are grateful to the ILP group at the Turing Institute. We are also thankful to Dale Miller and Frank Pfenning for providing general information in higher-order logic programming.

\section{A Syntax of $\lambda$-calculus terms}

A term in (simply typed) $\lambda$-calculus can be one of the following:

1. Atom. A variable or constant (of type $\alpha$ ) is a term (of type $\alpha$ );

2. Application. An application of $E$ (of type $\beta \rightarrow \alpha$ ) to $F$ (of type $\beta$ ) is a term $(E F)$ (of type $\alpha$ );

3. Abstraction. The abstraction of a term $F$ (of type $\alpha$ ) on a variable $X$ (of type $\beta$ ) is the term $(\lambda X . F)$ (of type $\beta \rightarrow \alpha$ ) that binds $X$ in the scope $F$.

The type of a term $F$ is denoted by $\tau(F)$. One may verify that (1), (2) and (3) in Section 1 are terms, and $\tau(P)=(\alpha, \alpha \rightarrow \beta)$ and $\tau(\leftarrow)=(\beta, \beta, \beta \rightarrow \gamma)$, assuming that $\tau(X)=\tau(Y)=\tau(Z)=\alpha$. Types are not essential in some results. They are polymorphic when used.

We denote $\left(\ldots\left(\left(\begin{array}{lll}F & \left.E_{1}\right) & \left.E_{2}\right)\end{array} \ldots E_{n}\right)\right.\right.$ by the expression $F\left(E_{1}, E_{2}, \ldots, E_{n}\right)$ if $F$ is an atom, and its type $\left(\alpha_{1} \rightarrow\right.$ $\left.\left(\alpha_{2} \rightarrow \ldots\left(\alpha_{n} \rightarrow \beta\right) \ldots\right)\right)$ by $\left(\alpha_{1}, \alpha_{2}, \ldots, \alpha_{n} \rightarrow \beta\right)$, where $\tau\left(E_{i}\right)=\alpha_{i} . F$ is called the functor, $E_{i}(i=1,2, \ldots, n)$ the arguments and $n$ the arity of $F$. We also abbreviate $\left(\lambda X_{1} .\left(\lambda X_{2} \ldots\left(\lambda X_{n} . F\right) \ldots\right)\right)$ to $\left(\lambda X_{1} X_{2} \ldots X_{n} . F\right)$ and $X_{1} X_{2} \ldots X_{n}$ to $\tilde{X}$.

The order of an atom is the depth of the nesting of parentheses in its type +1 . The order of a term is the highest order of its atoms. The order of $X, Y$ and $Z$ in (3) is 1 , and the order of $P$ is 2 . The order of (3) is therefore 2.

\section{A.1 $\lambda$-conversions}

Let $F\{X / E\}$ be the operation of substitution that replaces each occurrence of $X$ in $F$ by $E . X$ is free in $F$ if it does not occur in the scope of an abstraction in $F$ that binds $X . E$ is free for $X$ in $F$ if $E$ does not appear in the scope of an abstraction in $F$ that binds $X$. These two conditions are used to avoid possible name clashes in the following definition of $\lambda$ conversions.

A substitution is a set of ordered pairs $\theta=\left\{X_{i} / E_{i} \mid i=\right.$ $1, \ldots, n\}$, where $X_{i}$ are distinct variables and each $E_{i}$ is a term of the same type as $X_{i}$. The application of $\theta$ to a term $F$ is denoted by $F \theta$ and it is the term $\left.\left.\left(\ldots\left((\lambda \tilde{X} . F) E_{1}\right) E_{2}\right) \ldots\right) E_{n}\right)$. Intuitively for each $X_{i}(i=1, \ldots, n) F \theta$ is the results of replacing $X$ at each place in $F$ by the subterm $E_{i}$. The composition of substitutions, denoted by $\theta . \delta$, is the same as defined in first-order logic (sometimes we may omit the dot).

The conversion rules of the general $\lambda$-calculus is:

1. $\alpha$-rule. $\lambda X . F$ to $\lambda Y .(F\{X / Y\})$ if $Y$ is free for $X$ in $F$, and vice versa;

2. $\beta$-rule. $(\lambda X . F) E$ to $F\{X / E\}$ if $E$ is free for $X$ in $F$, and vice versa;

3. $\eta$-rule. $\lambda X . F X$ to $F$ if $X$ is not free in $F$, 
and vice versa.

The convertibility ${ }^{1}$ of two terms is an equivalence relation. The rules can be carried out in both left-to-right and right-to-left directions. $\lambda$-Convertible terms are considered to be equal to each other. An application of a conversion rule is called a $\alpha$ (or $\beta$ and etc.) $r e-$ duction when applied in the left-to-right direction and an expansion in the opposite direction. A $\lambda$ term that cannot be reduced by rules of any kind is said to be in normal form.

Church and Rosser proved that the normal form of a term is unique. For any convertible $\lambda$ terms $E$ and $F$, there is a term $S$ in normal form such that $E$ and $F$ can be reduced to $S$. They also proved the normal form of a term can be obtained by attacking the leftmost reduction on one-by-one basis until no reduction is possible. We should bear in mind at this point, apart from $\alpha, \beta$ and $\eta$ rules, there are other conversion rules that also maintain Church and Rosser properties such as the $\delta$ and $\delta_{0}$ rules that will be introduced in Appendix B.

\section{B Extension to $\delta_{0}$ conversion}

We will first consider a conversion rule of $\delta_{0}$, which is a variant of the $\delta$ conversion rule studied by Church in an extension to $\alpha \beta \eta \lambda$-calculus. The $\delta_{0}$ conversion rule is defined as follows. If $E$ and $F$ are in $\beta \delta_{0} \eta$ normal form without variables free in $E$ and $F$ :

1. $\delta_{0} E F=\lambda X Y . X$ if $E$ is $\alpha$-convertible to $F$;

2. $\delta_{0} E F=\lambda X Y . Y$ otherwise.

$\lambda X Y . X$ is known as "true" and $\lambda X Y . Y$ as "false" in the standard $\lambda$-calculus (In Church's system, truth is expressed by $\lambda X Y . X Y$ - the combinatory number 1 - and falsity by $\lambda X Y . X(X Y)$ - the number 2 . This is an arbitrary choice).

$\delta_{0}$ rule is similar to a weaker form of the equality theory introduced in modern deductive logic. Similarly to $\alpha \beta \eta \lambda$-calculus, the application of the $\delta_{0}$ rule from left-to-right is called a $\delta_{0}$ reduction. It is shown that $\delta_{0}$ reductions maintain the Church-Rosser properties of $\lambda$-calculus when the reduction expressions are carried over from the original (perhaps not in $\beta \delta_{0} \eta$ normal form) terms. We shall restrict ourselves to such a $\lambda$-calculus.

The term $\lambda X Y . X$ selects the first argument of $X$ and $Y$, whereas $\lambda X Y . Y$ selects the second. Using the $\delta_{0}$ rule we can construct a term: $F=$

\footnotetext{
${ }^{1}$ Note often an $\alpha$ conversion is used prior to a $\beta$ conversion to change the names of bound variables.
}

$\left(\lambda X .\left(\left(\delta_{0} X N\right) E_{1}\right) E_{2}\right)$. When $F$ is applied to a term $M$ we obtain $F M=\left(\left(\left(\delta_{0} M N\right) E_{1}\right) E_{2}\right)$ which corresponds to "if $M$ is $\alpha$ convertible to $N$, then $E_{1}$ else $E_{2}$ ", where $\delta_{0} M N$ is the $\delta_{0}$ reduction expression and $E_{1}$ and $E_{2}$ are the terms applied upon by this reduction. $M, N$, $E_{1}$ and $E_{2}$ are in $\beta \delta_{0} \eta$ normal form. $M$ and $N$ contain no variables free in them. We shall denote $F$ by " $\lambda X$.if $X=N$ then $E_{1}$ else $E_{2}$ ". Clearly, $\left(\lambda X . E_{1}\right)$ is convertible to $\left(\lambda X\right.$ if $X=N$ then $E_{1}$ else $E_{2}$ ) if $E_{1}$ is $\alpha$-convertible to $E_{2}$.

\section{References}

[Burstall and Darlington1977]

R.M. Burstall and J. Darlington. A transformation system for developing recursive programs. Journal of the $A C M, 24(1), 1977$.

[Curry et al.1958] H. Curry, R. Feys, and W. Craig. Combinatory Logic (Volume I). North Holland, Amsterdam, Holland, 1958.

[Dietzen and Pfenning1989] S. Dietzen and F. Pfenning. Higher-order and modal logic as a framework for explanation-based generalization. In B. Spatz, editor, Proceedings of the sixth international workshop on machine learning. Cornell University, Ithaca, New York, pages 447-449. San Mateo, CA: Morgan Kaufmann, June 1989.

[Feng and Muggleton1991] Cao Feng and Stephen Muggleton. Least general generalisation in higher order logic. TIRM, The Turing Institute, George House, 36 NOrth Hanover St, Glasgow, UK, 1991.

[Harao1990] Masateru Harao. Analogical reasoning based on higher order unification. In First International Conference on Algorithmic Learning Theory, pages 151-163, Tokyo, Japan, 1990. Japanese Society for Artificial Intellligence.

[Huet and Lang1978] G. Huet and B. Lang. Proving and applying program transformations expressed with second order patterns. Acta Informatica, 11:31-55, 1978 .

[Huet1975] G. Huet. A unification algorithm for typed $\lambda$-calculus. Theoretical Computer Science, 1:27-57, 1975.

[Kietz and Wrobelto appear] J. Kietz and S. Wrobel. Controlling the complexity of learning in logic through syntact ic and task orieted models. In S. Muggleton, editor, Proceedings of the First International Workshop on Inductive Logic Programming, Viana de Castelo, Portugal, to appear. Acadernic Press.

[Lavrac and Dzeroski1991] N. Lavrac and S. Dzeroski. Learning nonrecursive definitions of relations with 
linus. In Y. Kodratoff, editor, EWSL '91: machine learning: proceedings of the European working session on learning, pages 265-281, Porto, Portugal., 1991. Berlin: Springer-Verlag.

[McCarthy1960] J. McCarthy. Recursive functions of symbolic expressions and their computation by machine (part 1). Communications of the Association of Computational Machinery, 3:184-204, 1960.

[Miller1990] D. Miller. A logic programming language with $\lambda$-abstraction, function variables and simple unification. In P. Schroeder-Heister, editor, Extensions of logic programming, pages 237-258. SprinterVerlag LNCS, 1990.

[Muggleton and Feng1990] S. Muggleton and C. Feng. Efficient induction of logic programs. In First International Conference on Algorithmic Learning Theory, pages 369-381, Tokyo, Japan, 1990. Japanese Society for Artificial Intellligence.

[Muggleton1990] S. Muggleton. Inductive logic programming. In First International Conference on Algorithmic Learning Theory, volume Also in New Generation Computing, 1991, Vol 8, pages 42-61, Tokyo, Japan, 1990. Japanese Society for Artificial Intelligence.

[Nadathur and Miller1990] G.

$\mathrm{Na}-$ dathur and D. Miller. Higher-order horn clauses. Journal of the Association for Computing Machinery, 37(4):777-814, 1990.

[Paulson1986] L. Paulson. Natural deduction as higher-order resolution. Journal of Logic Programming, 3(3):237-258, 1986.

[Pfenning1991] F. Pfenning. Unification and antiunification in the Calculus of Constructions. In G. Kahn, editor, Sirth Annual Symposium on Logic in Computer Science, pages $74-85$. IEEE, July 1991.

[Plotkin1970] G.D. Plotkin. A note on inductive generalisation. In B. Meltzer and D. Michie, editors, Machine Intelligence 5, pages 153-163. Elsevier North-Holland, New York, 1970.

[Plotkin1971] G.D. Plotkin. A further note on inductive generalisation. In B. Meltzer and D. Michie, editors, Machine Intelligence 6, pages 101-124. Elsevier North-Holland, New York, 1971.

[Quinlan1990] J.R. Quinlan. Learning logical definitions from relations. Machine Learning, 5(3):239266, 1990.

[Raedt and Bruyonooghe1990] L. De Raedt and M. Bruyonooghe. Constructive induction by analogy: a new method to learn how to learn? In K. Morik, editor, EWSL'89: proceedings of the fourth european working session on learning, Montpellier, France, 1990. London: Pitman.

[Reynolds1970] J.C. Reynolds. Transformational systems and the algebraic structure of atomic formulas. In B. Meltzer and D. Michie, editors, Machine Intelligence 5, pages 135-151. Elsevier North-Holland, New York, 1970. 